\title{
Séparation isotopique par laser
}

\author{
D. Doizi
}

CEA, CE Saclay, DCC/DPE/SPL/SCLO, Bât. 467, 91191 Gif-sur-Yvette cedex, France

L'enrichissement de l'Uranium consiste à augmenter la teneur de l'isotope $235(0,7 \%$ dans l'uranium naturel) jusqu'aux teneurs utilisables dans les réacteurs produisant l'électricité (entre 3 et $5 \%$ ) en rejetant un produit très appauvri de cet isotope (moins de $0,2 \%$ ).

Cette activité importante intervient pour environ $10 \%$ dans le coût du $\mathrm{kWh}$ d'origine électronucléaire c'est -à- dire pour environ 2 centimes.

Exprimée en termes de marché mondial, l'activité enrichissement représente environ 25 milliards de francs.

Les besoins mondiaux s'élèvent à environ 30 MUTS/an (I'UTS est l'Unité de Travail de Séparation, on retiendra que 10 UTS sont nécessaires pour produire un kg d'Uranium enrichi à $5 \%$ à partir de $10 \mathrm{~kg}$ d'Uranium naturel). Ils sont couverts principalement par les procédés de diffusion gazeuse et d'ultracentrifugation.

L'usine de diffusion gazeuse Georges Besse située dans la Drôme a une capacité de 10,8 MUTS/an, elle couvre le quart des besoins mondiaux.

Le marché mondial se caractérise aujourd'hui par une surcapacité de production. A l'horizon 2010 , les usines actuelles de diffusion gazeuse très consommatrices en énergie $(2450 \mathrm{kWh}$ par UTS) devront être remplacées par des usines nouvelles permettant un abaissement très significatif du coût de l'UTS.

Le procédé SILVA (Séparation Isotopique par Laser sur Vapeur Atomique) est le procédé étudié en France qui pourrait se substituer avantageusement à la diffusion gazeuse. Ce procédé, qui utilise une excitation résonnante de l'Uranium 235, se caractérise par une séparation effectuée en une étape (1400 étapes pour la diffusion gazeuse), une consommation énergétique environ 25 à 40 fois moindre, une taille environ dix fois plus petite et un objectif de coût environ moitié de celui de la diffusion gazeuse amortie.

Le CEA y consacre, en collaboration avec le futur opérateur industriel COGEMA, environ 427 millions de francs cette année.

Le procédé SILVA est composé de deux grands sous-ensembles :

- le séparateur qui génère la vapeur atomique,

- l'atelier laser qui fournit les photons permettant l'ionisation sélective de l'Uranium 235.

Un schéma de principe est représenté sur la figure 1.

Ces deux entités seront décrites successivement en insistant plus particulièrement sur l'atelier laser, objet de cette présentation.

\section{SOUS ENSEMBLE SÉPARATEUR}

Le séparateur est une enceinte destinée à fournir la vapeur atomique d'Uranium. Il comprend un 
creuset, refroidi extérieurement à l'eau, contenant le lingot d'Uranium qui est vaporisé à $3000^{\circ} \mathrm{C}$ sous l'action d'un faisceau d'électrons. Ce faisceau est délivré par un canon à électrons qui peut être à cathode linéaire ou à balayage. Le jet de vapeur d'Uranium émis interagit avec les photons laser dans des zones d'irradiation où s'effectue l'ionisation sélective de l'Uranium 235.

Ce dernier, une fois ionisé, est attiré sur des plaques de collectage polarisées qui constituent le collecteur riche. Les atomes d' Uranium 238, non affectés par l'interaction laser, se condensent sur le dôme du séparateur appelé collecteur pauvre.

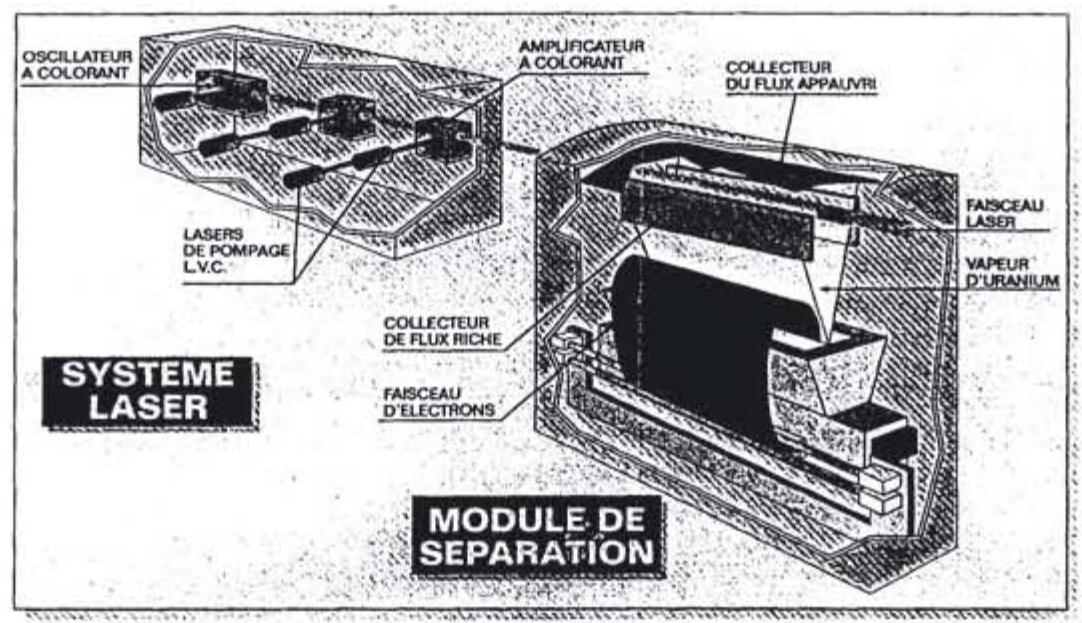

Figure 1

La gestion de l'Uranium s'effectue de manière liquide ce qui impose une température dans l'enceinte voisine de $1200^{\circ} \mathrm{C}$.

La capacité de production typique d'un tel ensemble est d'environ 100000 UTS/an soit la consommation annuelle d'une centrale à eau pressurisée de 1000 MWe. Il évapore de l'ordre de 150 tonnes d'Uranium par an.

La conception du séparateur doit prendre en compte le fonctionnement à haute température en présence d'un métal liquide corrosif et en présence de tensions électriques. Une activité importante de $R \& D$ a pour objet le choix des matériaux constitutifs de l'enceinte et leur tenue à la corrosion.

\section{Physique du procédé}

Le procédé utilise la différence entre les fréquences d'excitation des couches d'électrons des isotopes $U^{235}$ et $U^{238}$. L'ionisation sélective de $U^{235}$ (potentiel d'ionisation égal à $6,18 \mathrm{eV}$ ) est réalisée en trois étapes et quatre couleurs ainsi qu'il est montré sur le schéma sur la figure 2.

Le choix de la voie en trois étapes est dicté par le fonctionnement satisfaisant des lasers à colorant dans le domaine visible $(550-700 \mathrm{~nm})$. Une voie en deux étapes conduit en effet à une photodégradation importante des colorants utilisés.

Seules les deux premières étapes sont sélectives, les états auto-ionisants présentant une largeur de raie importante. La sélectivité du procédé est grande car elle est le produit des sélectivités des deux premières étapes. Le schéma d'ionisation utilise une quatrième couleur car, compte tenu de la température élevée, les atomes d'Uranium sont aussi présents sur le niveau situé à $620 \mathrm{~cm}^{-1}$ du niveau fondamental. 


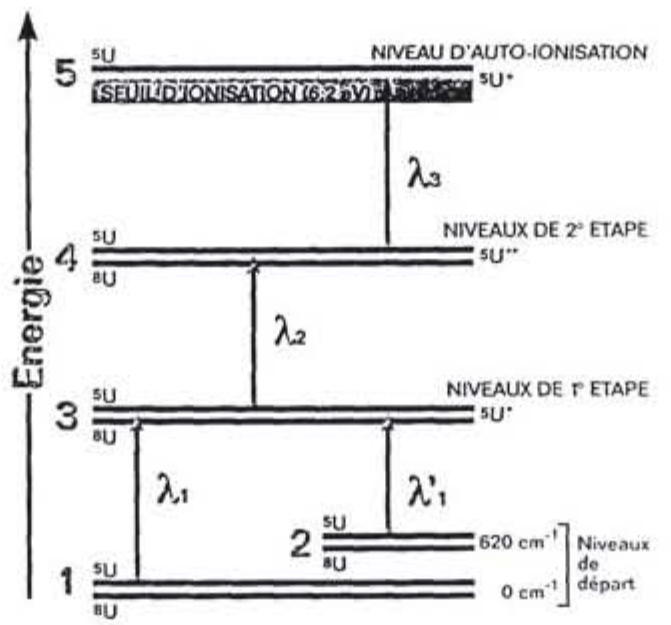

Figure 2

Le spectre d'absorption de l'Uranium est représenté sur la figure 3.

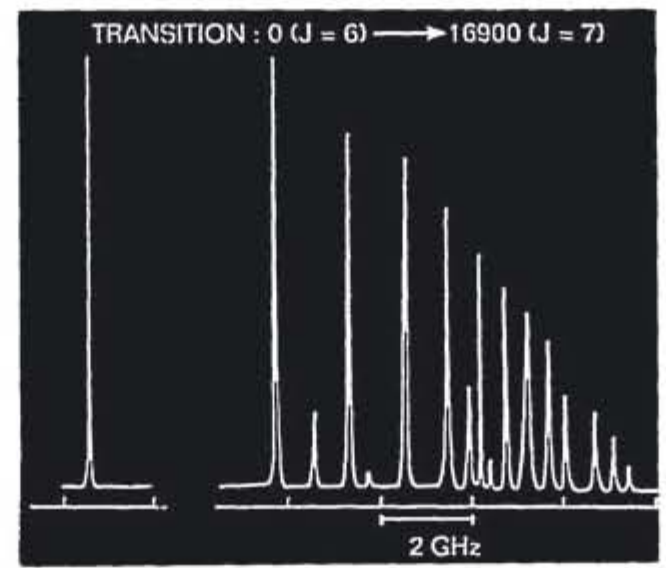

Figure 3

Les deux bandes sont séparées par l'écart isotopique. $\mathrm{L}^{\prime} \mathrm{U}^{235}$ présente une structure hyperfine qui élargit la raie. Un travail de spectroscopie important est donc nécessaire pour déterminer le meilleur plan-type (meilleure combinaison de longueurs d'onde) conduisant à de bons écarts isotopiques et à des structures hyperfines pas trop larges. Cette combinaison de longueurs d'onde est réalisée par le multiplexage spectral de lasers à colorant pompés optiquement par des Lasers à Vapeur de Cuivre (LVC).

Les caractéristiques souhaitées pour les lasers sont fixées par la technico-économie du procédé. 
Ce sont :

- l'accordabilité : le choix d'un procédé à quatre couleurs nécessite de délivrer des longueurs d'onde précises dans un domaine spectral large $(550-700 \mathrm{~nm})$.

- largeur spectrale : les deux premières étapes étant sélectives, elles demandent une largeur spectrale faible. Néanmoins, cette largeur ne peut être trop faible pour couvrir toute la largeur de la structure hyperfine de I'U $U^{235}$.

- cadence de répétition : le jet atomique de vapeur d'Uranium s'élève à une vitesse d'environ $1000 \mathrm{~m} / \mathrm{s}$. L'irradiation de tous les atomes d'U $\mathrm{U}^{235}$ présents dans la zone d'interaction nécessite des cadences de répétition laser d'au moins $10 \mathrm{kHz}$.

- durée des impulsions: Il faut que la durée de l'impulsion laser soit inférieure à la durée de vie radiative des états excités.

- énergie des impulsions: Pour utiliser au mieux les atomes produits, les faisceaux laser devront présenter des fluences permettant de saturer les transitions atomiques.

- rendement : la consommation d'énergie du laser ne doit pas dépasser celle qui est nécessaire pour produire les atomes d'Uranium.

Les lasers choisis, répondant à ces nombreux critères, sont les lasers à colorant pompés par les lasers à vapeur de cuivre (LVC).

\section{SOUS ENSEMBLE LASER}

\subsection{Description de la chaîne laser à colorant}

\section{Oscillateur à colorant :}

La chaîne laser à colorant est composée tout d'abord d'un oscillateur maître en configuration Hänsch qui génère un faisceau colorant entre 550 et $700 \mathrm{~nm}$. en utilisant trois colorants différents (rhodamines, mérocyanines,...). L'oscillateur est composée d'une cavité courte de longueur $30 \mathrm{~cm}$ composée d'un coupleur de sortie de transmission optimisée et d'un réseau en montage Littrow (autocollimation) servant de miroir de fond de cavité.

Une cellule à circulation contenant le milieu actif est excitée transversalement par le faisceau de pompage. La convolution du profil de la raie d'émission du colorant sélectionné par le réseau et du profil de la cavité nous donne un profil spectral dans lequel oscillent quelques modes longitudinaux. L'obtention d'une émission monomode longitudinale est obtenue en augmentant la sélectivité du laser par introduction d'un étalon de Fabry -Perot à lame d'air.

Des asservissements électroniques sur le mode (pour conserver le caractère monomode) et sur la longueur d'onde (permettant de conserver la fréquence optique) sont réalisés.

Un schéma de l'oscillateur à colorant est représenté sur la figure 4.

Les spécifications nominales d'un tel dispositif sont résumées sur le tableau 1.

Afin de couvrir la largeur de la raie de $\mathrm{I}^{\prime} \mathrm{U}^{235}$ (quelques $\mathrm{GHz}$ ), on balaie en fréquence l'impulsion de l'oscillateur à colorant (de largeur $50 \mathrm{MHz}$ ) à l'aide d'un modulateur électrooptique.

Le faisceau colorant est ensuite envoyé dans une série d'amplificateurs à colorant qui ont pour seul objet d'augmenter la puissance du faisceau colorant sans modifier ses caractéristiques intensives. Le schéma général d'un amplificateur de puissance est montré sur la figure 5.

La définition d'un amplificateur à colorant nécessite :

- le dimensionnement du volume actif

- la connaissance de l'hydrodynamique de l'écoulement

- la prise en compte des contraintes optiques.

Dimensionnement du volume actif :

Le dimensionnement du volume actif dépend de la longueur d'onde à délivrer donc du choix du colorant. Nous avons développé un code de calcul basé sur les équations cinétiques d'évolution des populations et sur les équations de transport des flux de photons. Ce code, validé 
expérimentalement, permet de connaître le gain d'un amplificateur pompé biface transversalement en fonction de l'énergie de pompage et de l'énergie colorant injectée. Il prend en compte l'émission spontanée amplifiée et inclut l'absorption des états excités, facteur important qui diminue le gain du laser. Il utilise des données photophysiques et photochimiques du colorant telles que les sections efficaces d'absorption et d'émission du colorant, la durée de vie de fluorescence, le rendement quantique de fluorescence..
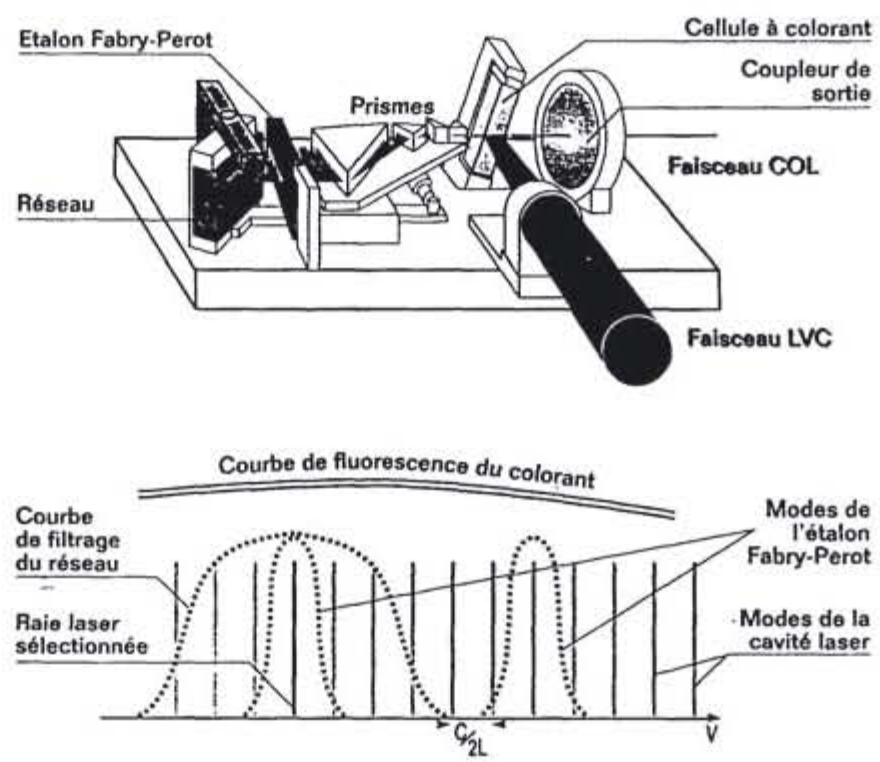

Figure 4

- STABILITE EN FREQUENCE :

- A LONG TERME $<50 \mathrm{MHz}$

- A COURT TERME $<10 \mathrm{MHz}$

- DOMAINE D'ACCORDABILITE : $550 \mathrm{~nm}-700 \mathrm{~nm}$

- DIVERGenCE : Limitee par LA DifFraction

- LaRgeur de RaIe : - $50 \mathrm{MHz}$

- ENERGIE DE L'IMPULSION : $-25 \mu \mathrm{J}$

- CADENCE DE REPETITION : supérieure â $5 \mathrm{kHz}$

- LARgeur d'TMPULSion : $-50 \mathrm{~ns}$

Tableau 1 


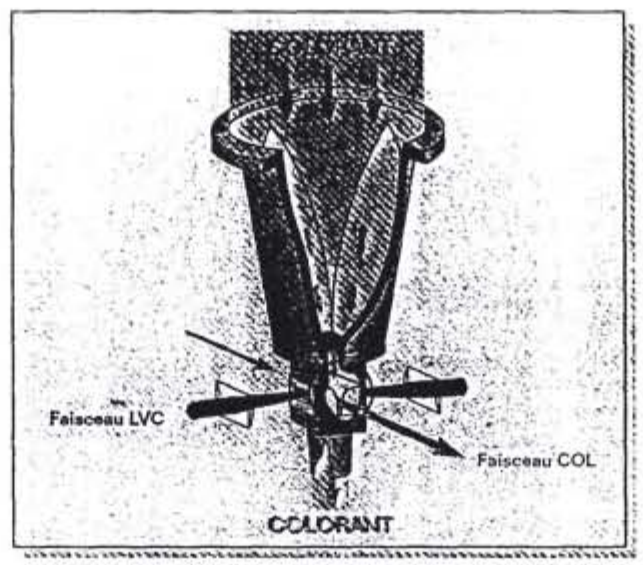

Figure 5

L'obtention de ces données est réalisée grâce à des bancs de mesure dont nous disposons.

Afin d'améliorer le rendement de conversion des amplificateurs à colorant, paramètre technico-économique de première importance, nous avons entrepris des travaux de synthèse organique.

Nous avons, par exemple, modifié la structure chimique de molécules commerciales pour les adapter à nos besoins.

Nous développons actuellement un code de modélisation des relations structure-propriétés utilisant des méthodes semi-empiriques pour orienter la synthèse organique.

Nous nous intéressons à plusieurs familles de molécules qui présentent chacune des caractéristiques particulières (xanthènes, mérocyanines, sels de xanthylium).

Notre objectif est l'obtention d'un rendement de conversion supérieur à $50 \%$ à chacune des quatre longueurs d'onde que nous devons délivrer.

\section{Hydrodynamique de l'écoulement :}

Le dimensionnement du volume actif ne conduit pas aux caractéristiques géométriques de l'amplificateur. Il faut tenir compte de l'hydrodynamique de l'écoulement. Ainsi, nous avons réalisé une veine optique spéciale permettant l'obtention d'un écoulement isotrope à fort nombre de Reynolds et échelle de turbulence réduite. Un soin particulier a été apporté à la suppression des zones de recirculation et au développement des couches limites.

Des essais en similitude Reynolds ont été réalisés sur une cellule grandie 40 fois.

Associé à cette veine, nous avons dimensionné le groupe de pompage. Celui-ci doit prendre en compte la perte de charge liée à la traversée de l'amplificateur, la cadence de répétition du laser de pompage et le débit à assurer.

Le groupe circulateur est un circuit bouclé, pressurisé, étanche, thermostaté. Il se compose d'une pompe (centrifuge ou volumétrique), d'un accumulateur surpresseur (destiné à supprimer la cavitation), d'un échangeur et d'un groupe froid (la température du colorant est régulée à $0,1^{\circ} \mathrm{C}$ près). Cet effort important de conception, associé à un choix des matériaux, permet de s'affranchir des problèmes liés à la lentille thermique et au marquage de dioptres. 


\section{Couplages optiques :}

Afin d'extraire complètement et proprement l'énergie stockée dans l'amplificateur, il est important de coupler optiquement les amplificateurs entre eux. Un code de diffraction permet la modélisation des différents couplages optiques possibles. Ce code prend en compte le phénomène d'amplification et les contraintes optiques associées au choix des matériaux. Le code est validé expérimentalement sur une chaîne laser d'étude où sont réalisées des mesures de phase et d'intensité du front d'onde colorant. Un exemple de résultat théorique et expérimental obtenu sur l'intensité du faisceau colorant en sortie d'amplificateur de puissance est montré sur la figure 6.

Des essais sont en cours sur le développement de techniques actives de correction de front d'onde.

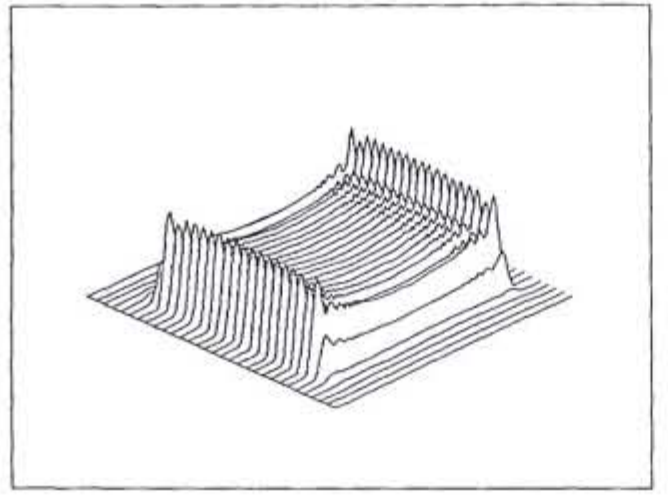

CALCULUTED INTENSITY AT THE EXIT OF THE POWER AMPLIFIER

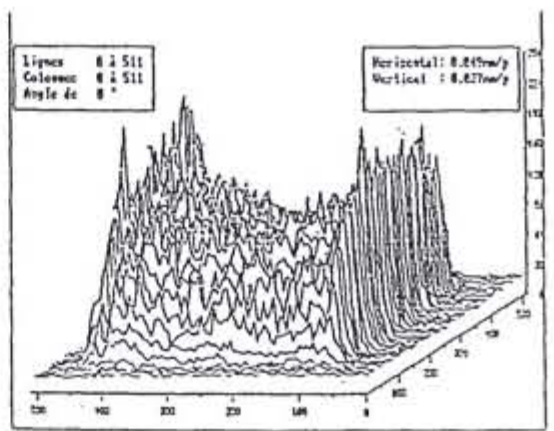

INTENSITY of a real beam (CCD camera + image analysis)

Figure 6

Les spécifications nominales obtenues pour la chaîne laser à colorant sont indiquées sur le tableau 2.

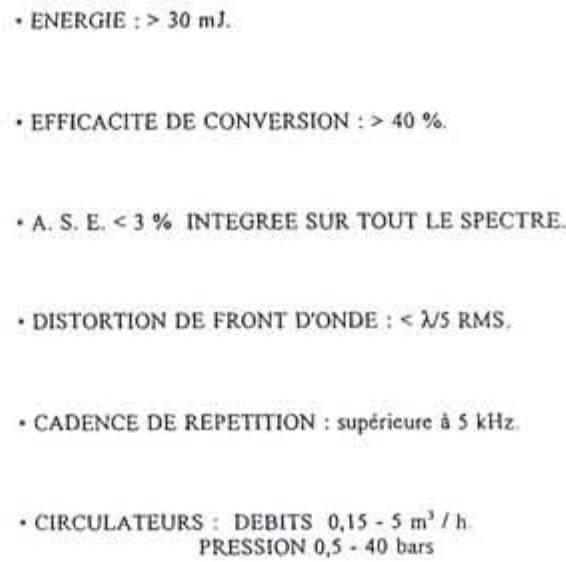

Tableau 2 


\subsection{Lasers de pompage}

a) solution de référence:

Le laser utilisé pour le pompage optique des lasers à colorant est le laser à vapeur de cuivre (LVC). Ce laser utilise une décharge axiale dans une vapeur de cuivre à $1500^{\circ} \mathrm{C}$. Ses caractéristiques principales sont :

- l'émission de deux longueurs d'onde 510,6 et 578,2 nm,

- une cadence de répétition élevée (voisine de $5 \mathrm{kHz}$ qui peut être augmentée par multiplexage temporel),

- une durée d'impulsion voisine de 50ns,

- une énergie par impulsion pouvant atteindre $200 \mathrm{~mJ}$.

Les atomes de cuivre neutre sont portés vers un niveau supérieur excité par collisions inélastiques avec les électrons produits dans une décharge impulsionnelle. Ils se désexcitent en émettant deux longueurs d'onde, les niveaux d'arrivée sont métastables et expliquent le fonctionnement pulsé du laser. Le LVC est un laser à 3 niveaux.

Le schéma spectroscopique est représenté sur la figure 7.

Cette décharge a lieu dans un mélange composé de gaz rare (néon, 30 torrs) et d'une faible pression partielle de cuivre à l'état de vapeur (environ 0,1 torr à $1420^{\circ} \mathrm{C}$ ou $3.10^{15}$ atomes $/ \mathrm{cm}^{3}$ ) Elle se produit entre deux électrodes cylindriques et est confinée dans un tube d'alumine de diamètre compris entre $30 \mathrm{~mm}$ et $80 \mathrm{~mm}$ et de longueur proche de $3 \mathrm{~m}$ suivant la puissance du laser. L'énergie volumique d'un tel laser est faible environ $10 \mu \mathrm{J} / \mathrm{cm}^{3}$. L'isolation thermique est assurée par un feutre d'alumine qui entoure le tube. L'enceinte externe permet le retour du courant avec une inductance minimale.

Le circuit électrique comprend une source continue haute tension de quelques dizaines de kV, un circuit à charge résonnante et un dispositif de commutation.

Les principaux travaux menés sur ces lasers consistent à obtenir les puissances les plus élevées avec le meilleur rendement possible (actuellement $400 \mathrm{~W}$ avec un rendement de 1,5\%), d'améliorer la durée de vie du laser en cherchant par exemple de nouveaux systèmes de commutation du type thyristors (qui remplacent les thyratrons) ou en augmentant la durée de vie de la charge de cuivre (environ 500 heures actuellement).

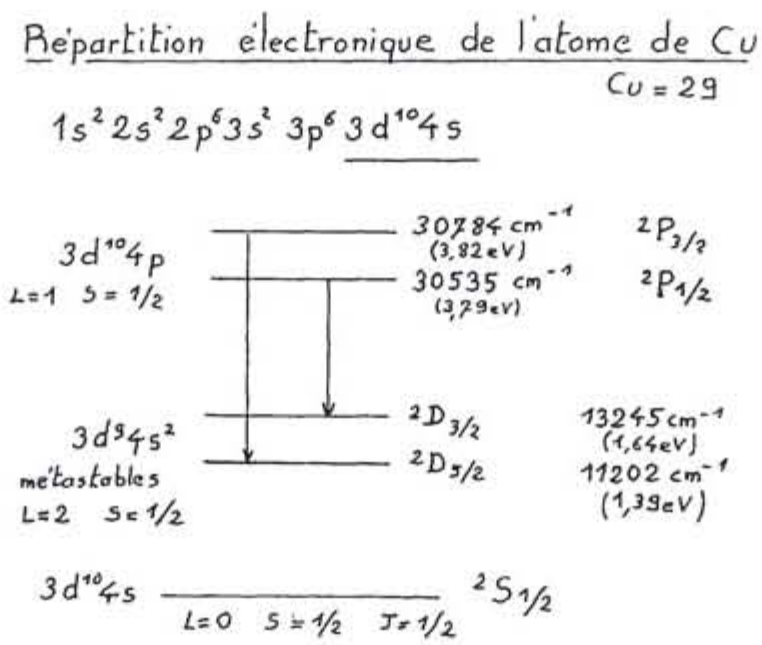

Figure 7 
Des travaux optiques concernent aussi l'amélioration de la qualité optique du faisceau par un choix judicieux de la cavité laser. A cause du gain élevé, le faisceau effectue peu d'aller-retour dans la cavité et la divergence obtenue en cavité stable plan concave atteint 5 mrad.

Les LVC sont généralement associés en chaîne comprenant un oscillateur maître (monté en cavité instable injectée permettant l'obtention d'un faisceau de divergence voisine de $250 \mu \mathrm{rad}$ ) suivi de trois ou quatre amplificateurs de puissance.

b) Solutions alternatives de pompage :

\section{Laser $\mathrm{Cu}-\mathrm{HBr}$}

Ce laser est un laser à vapeur de cuivre développé récemment à l'Université de St Andrews en Ecosse. Il utilise du bromure d'hydrogène $\mathrm{HBr}$ comme gaz réactif pour former $\mathrm{CuBr}$ en phase gazeuse partiellement dissocié par la décharge.

Les principaux avantages obtenus avec ce type de laser sont :

- une température de fonctionnement basse comprise entre 400 et $600^{\circ} \mathrm{C}$ (au lieu de $1500^{\circ} \mathrm{C}$ ) LVC)

- un temps de préchauffage court, voisin de 20 minutes (contre plus de deux heures pour le

- une cadence de répétition élevée, voisine de $20 \mathrm{kHz}$ (la recombinaison du plasma est plus rapide car le milieu est plus froid, présence d'une espèce fortement électronégative $\mathrm{Br}$ supplémentaire qui favorise la désexcitation des métastables)

- une résistivité du plasma plus élevée (le rendement de ce laser peut dépasser 3\%)

- un gain maximal au centre permettant l'obtention d'un profil gaussien stable en champ lointain (le LVC possède un maximum de gain à la paroi).

Tous ces avantages font du $\mathrm{Cu}-\mathrm{HBr}$ un candidat potentiel pour remplacer le LVC mais les problèmes inhérents aux lasers à gaz tels que l'encombrement demeurent.

Une inconnue doit aussi être levée qui concerne la corrosion liée à la présence de $\mathrm{HBr}$.

\section{Laser à solide}

Il s'agit d'un laser comprenant un milieu actif solide pompé optiquement et placé dans une cavité optique. Le laser Nd:YAG (grenat d'Yttrium et d'Aluminium dopé avec environ $1,1 \%$ en poids d'ions Néodyme) pompé par diodes laser ou par lampes et qui émet dans l'infrarouge à $1064 \mathrm{~nm}$ est un bon exemple.

Par comparaison aux LVC, les lasers à solides possèdent un grand nombre d'atouts :

- démarrage instantané : en effet, aucun temps de chauffe n'est nécessaire.

- système compact : il s'agit ici d'un avantage lié au génie civil de l'usine.

- durée de vie longue : le principal avantage certainement lié à cette technologie. En effet le LVC nécessite une maintenance importante (préventive ou curative) qui conduit à des coûts de fonctionnement importants. L'utilisation de lasers à solides pompés par des diodes laser permettrait l'obtention de temps moyen de bon fonctionnement (MTBF) proches de 10000 heures.

- le rendement de ces lasers est comparable à celui des LVC mais l'alimentation des diodes laser est du type basse tension fort ampérage $(2 \mathrm{~V}, 30 \mathrm{~A}$ pour une diode de $20 \mathrm{~W})$.

- le principal inconvénient associé à ce type de laser demeure les effets thermiques et leurs conséquences liés à un fonctionnement à haute cadence.

Le choix du pompage du milieu actif par des diodes laser permet de diminuer par un facteur proche de 3 les effets thermiques car l'émission des diodes laser (voisine de $810 \mathrm{~nm}$ ) est bien centrée sur les bandes d'absorption du néodyme.

Pour la conception de notre laser, nous avons opté pour un pompage optique continu d'un barreau de NdYAG au moyen de 15 barrettes de diodes émettant $20 \mathrm{~W}$ continus (modèle SDL3460-S).

Une puissance de $275 \mathrm{~W}$ collimatée est disponible à $810 \mathrm{~nm}$.

Une puissance de $82 \mathrm{~W}$ est obtenue en continu à $1064 \mathrm{~nm}$

Quand la cavité est déclenchée au moyen d'un dispositif acousto-optique, à $10 \mathrm{kHz}$, une puissance de $30 \mathrm{~W}$ est obtenue à $1064 \mathrm{~nm}$ en $115 \mathrm{~ns}$. 
Par insertion d'un cristal non linéaire de KTP de dimensions $3 \times 3 \times 5 \mathrm{~mm}$ dans la cavité qui permet le doublement de fréquence, une puissance de $14 \mathrm{~W}$ à $532 \mathrm{~nm}$ et $7,2 \mathrm{kHz}$ en $95 \mathrm{~ns}$ est obtenue.

Le dispositif expérimental réalisé est représenté sur la figure 8 :

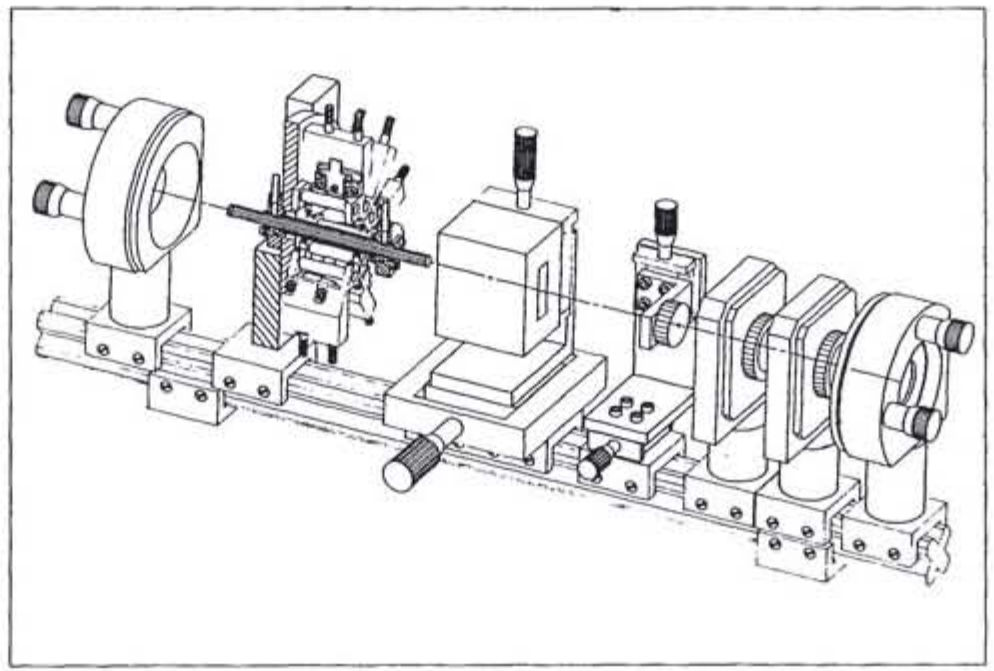

Figure 8

L'intérêt associé à ces lasers dépendra du coût du watt lumineux vert fourni en comparaison avec celui délivré par les LVC. La baisse très significative du prix des diodes laser de pompage constitue le point clé associé à cette technologie.

\section{CONCLUSIONS}

Nous avons donné ici un aperçu synthétique du procédé SILVA en décrivant plus particulièrement l'atelier laser. Les étapes - clés associées à ce projet sont :

- une démonstration technique réalisée en 1989 au niveau de quelques grammes d'Uranium enrichi.

- une démonstration complète prévue en 1996 - 1997 au niveau de quelques kilogrammes (Projet ASTER).

Cette démonstration associée à une étude technico-économique permettra de prendre une décision de poursuite du projet en vue de son industrialisation aux alentours des années 20052010. 